\title{
Synthesis and Characterization of New Hexahydro-1,3,5-S-Triazines Derivatives from Benzo Caine
}

\author{
Hayder Jawad Abed and Souad Jabbar Lafta \\ Chemistry Department, College of Science, Al-Mustansiriyah University, Baghdad, Iraq
}

Correspondence Author: Hayder Jawad Abed, Chemistry Department, College of Science, Al-Mustansiriyah University, Baghdad, Iraq E-mail: alfatlawichem@gmail.com; Tel: 009647715070768

Received date: April 2018, Accepted date: 28 July 2018, Online date: 5 August 2018

Copyright: (c) 2018 Hayder Jawad Abed and Souad Jabbar Lafta., This is an open-access article distributed under the terms of the Creative Commons Attribution License, which permits unrestricted use, distribution, and reproduction in any medium, provided the original author and source are credited.

\begin{abstract}
The synthesis of the hexahydro-1,3,5-s-triazine from the reaction of ethyl-p-aminobenzoate with aqueous formaldehyde $(37 \%)$ in absolute ethanol. hexahydro-1,3,5-s-triazine (A) is treated with hydrazine hydrate (99\%) in absolute ethanol gives the carbohydrazide (A1) The reaction of carbohydrazide (A1) with formic acid gives the amide derivative (A2) while its condensation with aromatic aldehydes in presence of glacial acetic acid as a catalyst afforded the schiff's bases $A(3-6)$. The treatment of hydrazide (A1) with aliphatic and aromatic anhydrides afforded the heterocyclic pyridazine derivatives A(7-9). Finally the reaction of the triazine $(A)$ with thiosemicarbazide in presence of Sodium acetate gives the compound (A10) which is treated with aromatic aldehydes to afford schiff's bases A(11-12).
\end{abstract}

Key words: triazin, Schiff's base, Pyridazines, Carbohydrazide, Thiocarbohydrazide.

\section{INTRODUCTION}

Hexahydro-1,3,5-s-triazines are six membered heterocyclic compounds consisting of three carbons and three nitrogen atoms, where the structure is symmetrical with alternating nitrogen and carbon atoms and with the six hydrogen atoms bonded to the carbon atoms. Hexahydro-1,3,5-s-triazines can be formed in a condensation reaction between a carbonyl and an amine compound (Katritzky A.R., 2002). The side group attached to the nitrogen atoms can then be chosen to influence the physical and chemical properties such as the solubility of the triazine. 1,3,5-hexahydro-s-triazine derivatives are reported to show a broad spectrum of biological activity in particular, antitumor, antimicrobial, and anticancer activities (Duden P. and M. Scharf, 1895). It is also used as herbicides, carcenolytics growth stimulating and antidote activity (Richter and W.P. Trautwein, 1969) 1,3,5-trinitrohexahydro-s-triazine is a highly explosive, white or gray powder that is often mixed with other explosives, oils or waxes to make military munitions and (Halasz, A. and J. Hawari, 2011) recently RDX has replaced TNT as a primary explosive in various munitions formulations. RDX which is classified by the US Environmental Protection Agency (USEPA) as a potential carcinogen is toxic to organisms (Rylott, E.L., 2010) rguably, triazines are the most common chemical scavenger used in the field for the removal of low level hydrogen sulfide and mercaptans from hydrocarbon stream (Petroleum) (Thomas, R.O. and D.C. Peter, 2010) Other uses as cross-linking agent for improving the fixation of dyes on polyamide fibers, (Lewis, D.M. and Y.C. Ho, 1995) screened biological activity, (Braulio, I., 2010) corrosion inhibitors, (Sudhish, K.S., 2012) antihypertensive activity and antiviral activity (Vinod, K.P., 2004).

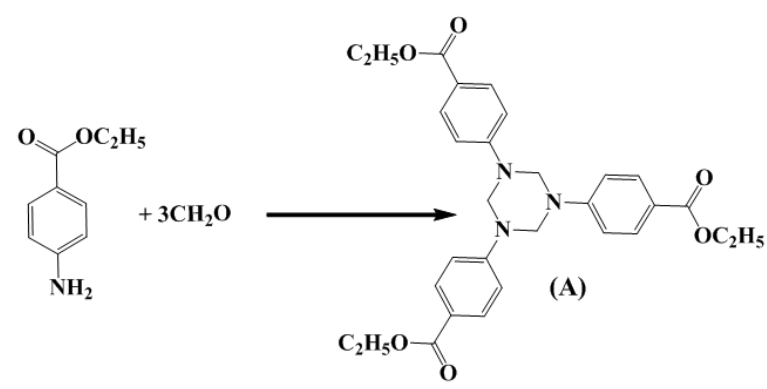

Schem1: synthesis of 1, 3, 5-triaryl-1, 3, 5-hexahydro-striazines-1,3,5triyl [A]

2.1. General procedure of the synthesis of 1, 3, 5-triaryl-1, 3, 5-hexahydro-s-triazines $(A)$ :

A mixture of ethyl-p-aminobenzoate $(10 \mathrm{mmol})$ and $(30 \mathrm{mmol})$ of aqueous formaldehyde solution $37 \%$ was dissolved in $20 \mathrm{~mL}$ absolute ethanol. The reaction mixture was stirred at room temperature for $0.5 \mathrm{hrs}$, the precipitated was left over night and washed with cooled ethanol, the product recrystallized from absolute ethanol. 


\subsection{Procedure of the synthesis of (1,3,5-triazinane-1,3,5-triyl)tri(benzohydrazi-de) (A1):}

A mixture of compound (A) $(0.53 \mathrm{gm}, 1 \mathrm{mmol})$ and $5 \mathrm{~mL}$ of hydrazine hydrate $99 \%$ in $15 \mathrm{~mL}$ absolute ethanol were heated under reflux for $36 \mathrm{hrs}$ (the reaction was monitored by TLC). After completion of the reaction, the mixture was cooled to room temperature and poured in crushed ice and the solid formed was filtered, washed with cold water and recrystallized from ethanol.

2.3. Procedure for the synthesis of (1,3,5-triazinane-1,3,5-triyl)tris( $N^{\prime}$-formylbenzohydrazide) (A2):

A solution of compound $(\mathbf{A 1})(0.49 \mathrm{gm}, 1 \mathrm{mmol})$ in formic acid $(0.13 \mathrm{gm}, 3 \mathrm{mmol})$ was refluxed for $3 \mathrm{hrs}$ (the reaction was monitored by TLC) in $10 \mathrm{ml}$ absolute ethanol, The reaction mixture was cooled and poured on distill water, The formed precipitate was filtered and recrystallized from absolute ethanol.

2.4.Procedure for the synthesis of 4,4',4"-(1,3,5-triazinane-1,3,5-triyl)tris( $N^{\prime}$-(arylidene)-benzohydrazide) A(3-6):

A solution of (A1), $(0.49 \mathrm{gm}, 1 \mathrm{mmol})$ and substituted aromatic aldehyde $(3 \mathrm{mmol})$ in methanol with addition of four drops of glacial acetic acid The mixture was subjected to M .W irradiation at 300 watts, $140 \mathrm{c}^{\circ}$ for 10 minutes (the reaction was monitored by TLC), After completion of reaction, the mixture was cooled and the formed solid separated on cooling, was filtered washed with cold methanol and recrystallized from the suitable solvent.

\subsection{General procedure for 1,1',1"-(4,4',4"-(1,3,5-triazinane-1,3,5-triyl)tris-(benzoyl))tris(1,2-dihydropyridazine-3,6-dione) A(7-9):}

A mixture of carbohydrazide (A1), $(1 \mathrm{mmol})$ and anhydride $(3 \mathrm{mmol})$, in $15 \mathrm{~mL}$ absolute ethanol and glacial acetic acid $(3 \mathrm{mmol})$ was refluxed for $9 \mathrm{hrs}$ (the reaction was monitored by TLC). After cooling the reaction mixture was poured on to crushed ice and the formed solid was filtered, washed with dilute Sodium bicarbonate solution and recrystallized from suitable solvent.

\subsection{Procedure of the synthesis of 1,3,5-triazinane-1,3,5-triyl)tris(benzoyl)) tris -(hydrazinecarbothiamide) (A10):}

A mixture of compound (A) $(0.53 \mathrm{gm}, 1 \mathrm{mmol})$ with thiosemicarbazide $(3 \mathrm{mmol})$ and sodium acetate $(3 \mathrm{mmol})$ in $(25 \mathrm{~mL})$ absolute ethanol was refluxed for 24 hrs (the reaction was monitored by TLC) The reaction mixture was poured on to ice cold water and the formed precipitate was filtered and recrystallized from absolute ethanol.
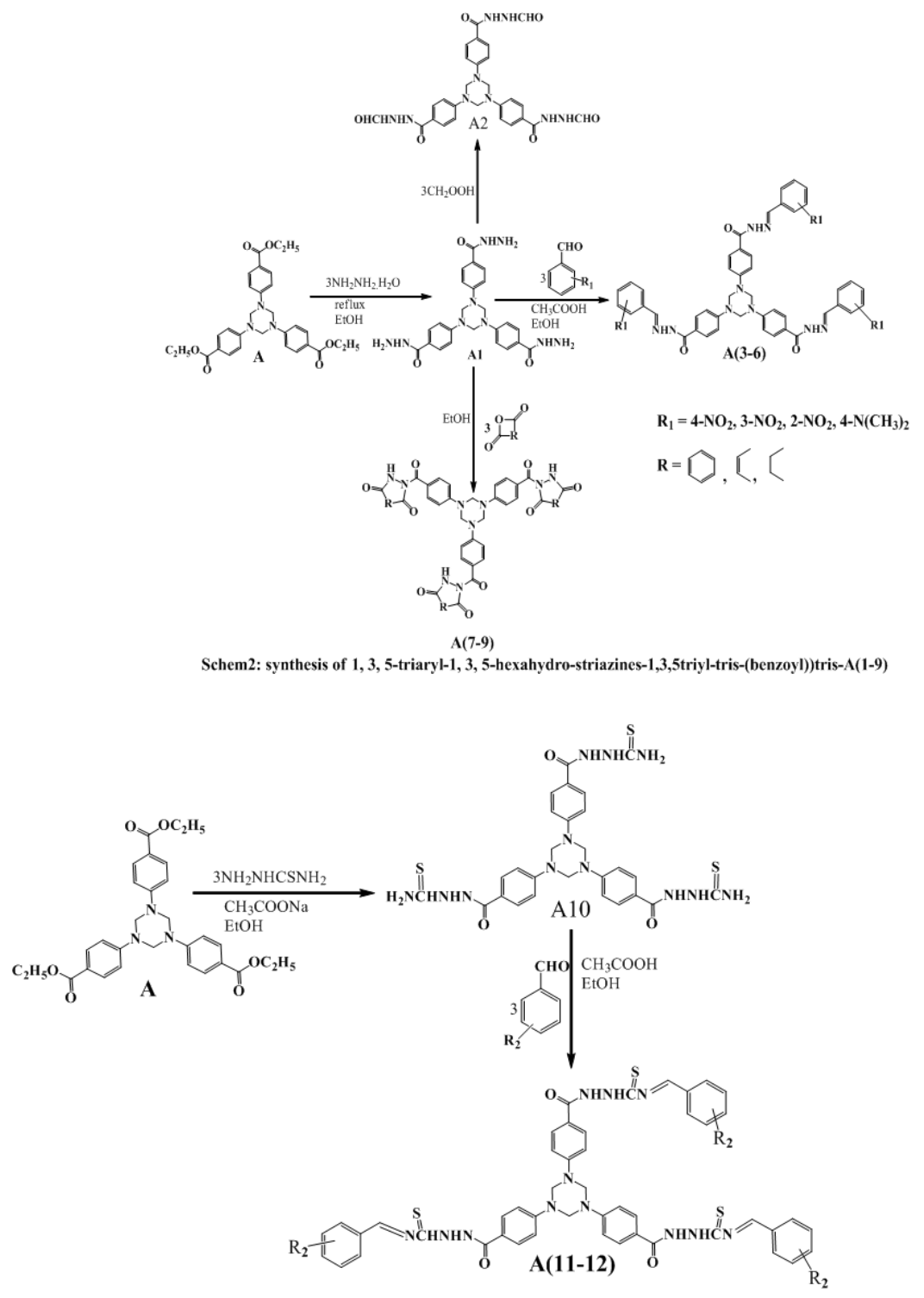

$\mathrm{R}_{2}=4-\mathrm{NO}_{2}, 3-\mathrm{NO}_{2}$,

Schem3: synthesis of (1,3,5-triazinane-1,3,5-triyl)tris(benzoyl))tris(N-(arylidene)hydrazinecarbothioamide

2.7.General procedure for the synthesis of (1,3,5-triazinane-1,3,5-triyl)tris(b-enzoyl))tris( $N$-(arylidene-)hydrazinecarbothioamide)A(11-12):

A solution of (A10) $(0.66 \mathrm{gm}, 1 \mathrm{mmol})$ and substituted aromatic aldehyde $(3 \mathrm{mmol})$ in methanol with addition of four drops of glacial acetic acid The mixture was subjected to M.W irradiation at 300 watts, 140 oc for 10 minutes, After completion of reaction, the mixture was cooled and the formed solid separated on cooling, was filtered washed with cold methanol and recrystallized from the suitable solvent. 


\section{Chemicals and Instruments:}

All chemicals are used throughout the work are (BDH- England), (Sigma \Ald-rich Germany), (Scharlau \Spain), (Himedia / India) origin .they are used without any purification. Melting points are determined in open capillary tubes in a Gallen Kamp MFB-600-Melting Point apparatus and are uncorrected. Infrared spectra were recorded directly in a SHIMADZU FT-IR 8400S,1H-NMR and 13C-NMR spectra (solvent DMSO-d6 or CDCl3) were recorded onBruker DMX-500 spectrophotometer $300 \mathrm{MHz}$ or $500 \mathrm{MHz}$ spectrometer with TMS as internal standard which were made at chemistry department, Ahl Al-Bayt University, Jordan. Some of the reactions were carried out by using microwave oven (Anton Paar) Monowave 300, Rotary Evaporator the solvents were evaporated by BUCHI Rotavapor R-205, Thin layer chromatography the purity of the synthesized compounds was detected by (TLC) technique using silica gel as stationary phase and chloroform or (n-hexane: ethyl acetate) in different ratios as eluents.

\section{RESULTS AND DISCUSSION}

Triazine derivatives were prepared according to method reported in the synthetic scheme.

4.1.(A) White, 79\%, m.p.192c $\mathrm{c}$, FT-IR(cm-1) spectrum of compound (A) shows disappearance of stretching band of (NH2) group at frequency (3421,3340) $\mathrm{cm}-1$ and appearance of stretching band at frequency $(1681) \mathrm{cm}-1$ for $(\mathrm{C}=\mathrm{O})$ group.1H-NMR spectrum of compound $(\mathrm{A})$ shows: $\delta=1.2 \mathrm{ppm}(\mathrm{t}, 9 \mathrm{H}, 3 \mathrm{CH} 3)$, $\delta=4.1 \mathrm{ppm}(\mathrm{q}, 6 \mathrm{H}, 3 \mathrm{CH} 2), \delta=4.5 \mathrm{ppm}(\mathrm{s}, 3 \mathrm{H},(\mathrm{N}-\mathrm{CH}-\mathrm{N})$ axial) $\delta=6.7 \mathrm{ppm}(\mathrm{d}, 6 \mathrm{H}, \mathrm{Ar}-\mathrm{H}) \delta=7.2 \mathrm{ppm}(\mathrm{S}, 3 \mathrm{H},(\mathrm{N}-\mathrm{CH}-\mathrm{N})$ equatorial) $\delta=7.2 \mathrm{ppm}(\mathrm{d}, 6 \mathrm{H}, \mathrm{Ar}-\mathrm{H}) . \mathrm{In}$ additional to all the above, the 13C-NMR spectrum of compound (A) showed the following signalsof carbon $\square \square \mathrm{ppm}$ : 14.8(CH3), 51.6(-N-CH2), 60(-O-CH2alph), 112(-CH2ar), 117(C-CO-), 131(CH2ar), 152(N-Car), 166.2(-C=O-).

4.2. (A1) White 79\%, m.p 83c,FT-IR cm-1: (3338) cm-1 for $(\mathrm{NH}),(3225,3421)$, $(\mathrm{NH} 2)$ and $(1680)(\mathrm{C}=\mathrm{O})$.

4.3.(A2) White, $80 \%$, m.p148c, FT-IR: $(\mathrm{cm}-1) 3317(\mathrm{~N}-\mathrm{H})$, and $1683(\mathrm{C}=\mathrm{O})$.

4.4. Characterization of compounds A(3-6):

4.4.1. (A3) yellow, 80\%, m.p. 190c, FT-IR: (cm-1) 3107(NH), 1701(C=O), 1629(C=N), 1514,1346(NO2).

4.4.2. (A4) Yellow, 77\%, m.p 161c, FT-IR: (cm-1) 3367(NH), 1701(C=O), 1631(C=N), 1531, $1365(\mathrm{NO} 2)$.

4.4.3. (A5) Orang-yellow 76\%, m.p 117c,FT-IR:(cm-1) 3363(NH), 1703(C=O), 1604(C=N).

4.4.5. (A5) Yellow 70\%, m.p 131c, FT-IR(cm-1) 3342(NH), 1680(C=O), 1600(C=N), 1519,1340(NO2).

5. Characterization of compounds A(7-9):

5.1.(A7) White, 77\%, m.p 172c, FT-IR:(cm-1) 3290(NH), 1710(C=O), 1641(C=Caromatic).

5.2. (A8)White 83\%, m.p 154c, FT-IR: (cm-1) 33429(NH), 1712(C=O), 1599(C=C aromatic).1HNMR of $(\mathrm{A} 8) \delta=2.6 \mathrm{ppm}(\mathrm{m}, 12 \mathrm{H}, 3 \mathrm{CH} 3), \delta=4.3 \mathrm{ppm}(\mathrm{s}, 6 \mathrm{H},-$ $\mathrm{NCH} 2 \mathrm{~N}-), \delta=6.7 \mathrm{ppm}(\mathrm{d}, 6 \mathrm{H}, \mathrm{Ar}-\mathrm{H}), \delta=7.9 \mathrm{ppm}(\mathrm{d}, 6 \mathrm{H}, \mathrm{Ar}-\mathrm{H}) \delta=10.3 \mathrm{ppm}(\mathrm{s}, 3 \mathrm{H}, \mathrm{NH})$

5-3-(A9) White 81\%, m.p198c, FT-IR:(cm-1) 3300(NH), 1705(C=O), 1614(C=Caromatic).

6. Characterization of compound (A10):

6.1. (A10) White,88\%, m.p 90c FT-IR: (cm-1)3346(NH), 3223, 3421(NH2), 1681(C=O)

6. Characterization of compounds A(11-12):

6.1. (A11) Yellow, 75\%, m.p 180c, FT-IR: (cm-1) 3113(NH),1697(C=O), 1629(C=N), 1527,1352(NO2).

6.2. (A12) Yellow, 70\%, m.p 160c, FT-IR: (cm-1) 3373(NH), 1701(C=O), 1631(C=N), 1514,1352(NO2).

Conclusion:

TheTriazinesderivativesin the manuscript have great potential as biological activityand many industrial uses

\section{ACKNOWLEDGEMENT}

The author wish to thank the all professors of Chemistry Department, College of Science, Al-Mustansiriyah University /Baghdad Iraq.

\section{REFERENCES}

Katritzky A.R., K. Suzuki and H.Y. He, 2002. J. Org. Chem, 67: 3109-3114.

Duden P. and M. Scharf, 1895. J.Ann., 288-218.

Richter and W.P. Trautwein, 1969. Chem. Ber, 102-931.

Halasz, A. and J. Hawari, 2011. ACS Symp. Ser. 10:_441-462.

Rylott, E.L., A. Lorenz and N.C. Bruce, 2010. Curr. Opin.Biotechnol., 17-22.

Thomas, R.O. and D.C. Peter, 2010. ASRL. Quarterly.Bulletin, 3: 1-21.

Lewis, D.M. and Y.C. Ho, 1995.Dyes. Pigm, 28: 171-192.

Braulio, I., G. Angelica, A. Rodring, N. Manuel and C. Justo, 2010. Molbank, 10: 667-664.

Sudhish, K.S., K.S. Ashish and M.A. Quraishi1, 2012. Int.J.Elctrochem. Sci., 7: 3371-3389.

Vinod, K.P., T. Sarah, T. Zehra, J. Madhawanand and B. Shashikala, 2004. J. Acta.Pharm., 54: 1-12. 